\title{
Reproductive outcome of male carriers of chromosomal abnormalities: multidisciplinary approach for genetic counseling and its implications
}

\author{
K.M. Guo ${ }^{1}$, B. Wu ${ }^{2}$, H.B. Wang ${ }^{3}$ and R.H. Tian ${ }^{4}$ \\ ${ }^{1}$ Department of Andrology, First Hospital of Jilin University, Changchun, \\ Jilin Province, China \\ 2Department of Psychology, Sixth People's Hospital of Changchun, \\ Jilin Province, China \\ ${ }^{3}$ Department of Pediatrics, First Hospital of Jilin University, Changchun, \\ Jilin Province, China \\ ${ }^{4}$ Department of Psychology, First Hospital of Jilin University, Changchun, \\ Jilin Province, China \\ Corresponding author: R.H. Tian \\ E-mail: tianrhjdyy@126.com
}

Genet. Mol. Res. 15 (4): gmr15048963

Received July 12, 2016

Accepted September 28, 2016

Published December 2, 2016

DOI http://dx.doi.org/10.4238/gmr15048963

Copyright $(92016$ The Authors. This is an open-access article distributed under the terms of the Creative Commons Attribution ShareAlike (CC BY-SA) 4.0 License.

\begin{abstract}
Chromosomal abnormality is the most common genetic cause of infertility. Infertility, as a psychological problem, has received an increasing amount of attention. Psychological interventions have been shown to have beneficial effects on infertile patients with chromosomal abnormalities. The present study explored reproductive outcome of male carriers of chromosomal abnormalities, who accepted genetic counseling and psychological support. Cytogenetic analysis
\end{abstract}


was performed using cultured peripheral blood lymphocytes and G-banding. The detection rate of chromosomal abnormalities was $10.3 \%$ in pre-pregnancy counseled males, with polymorphisms being most common, followed by 47,XXY and balanced translocation. Follow-up of 170 carriers with normozoospermia, after 3 years, showed that $94.7 \%$ of the cases resulted in live births. In the carriers of polymorphisms, balanced translocation, inv(9), Robertsonian translocation, inversion, and 47,XYY, live birth rates were 96.8, 85.7, $100,83.3,75$, and $100 \%$, respectively. Follow-up of 54 carriers with oligozoospermia or azoospermia, after 3 years, showed that $14.8 \%$ of the cases resulted in live births. In the carriers of 47,XXY with severe oligozoospermia or azoospermia, 80 or $5.9 \%$ of the cases resulted in live births, respectively. Therefore, timely psychological support would be beneficial and multidisciplinary approach should be preferentially considered for the management of individuals with chromosomal abnormalities.

Key words: Chromosomal abnormality; Reproductive outcome; Genetic counseling; Multidisciplinary approach; Infertility

\section{INTRODUCTION}

Chromosomal abnormality is the most common genetic cause of infertility (Zhang et al., 2015b). Male carriers of chromosomal abnormalities have often been found to be azoospermic or oligozoospermic (Naasse et al., 2015; Zhang et al., 2015b). Although balanced chromosomal forms exert no phenotypic effect on the carriers, they show variable influence on sperm counts, which can range from normal counts to oligozoospermia or even result in a total absence of sperm in the ejaculate (Zhang et al., 2015c). Hence, reproductive outcomes of the carriers often show normal fertility or infertility. For male carriers, the success rates of natural pregnancies range from 30 to 70\% (Ozawa et al., 2008). Nearly two-thirds of the carriers are likely to have a normal outcome in subsequent pregnancy (Kochhar and Ghosh, 2013), and cumulative live birth rate is $64.3 \%$ for couples in which the male has a chromosomal anomaly (Flynn et al., 2014).

For the carriers of chromosomal abnormalities, preventive care should be provided from the time of diagnosis, preferentially using a multidisciplinary approach, including clinical psychologist or psychiatrist, urologist, geneticist, sexologist, and a fertility team (Gies et al., 2014). Infertility, as a psychological problem, has received an increasing amount of attention (Zhang et al., 2015b). Psychological distress in carriers of chromosomal abnormalities should be taken seriously (Spitczok von Brisinski, 2013; Wang et al., 2016). Severe depressive symptoms are significantly associated with increased infertility-related distress at individual and partner levels (Peterson et al., 2014). Psychological interventions have beneficial effects on infertile patients with chromosomal abnormalities (Cousineau and Domar, 2007).

For counseling individuals with chromosomal abnormalities, accurate information and assessment of associated medical conditions should be offered at diagnosis and should be followed by psychological counseling (Gies et al., 2014; Zhang et al., 2015a; Wang et al., 2016). In this study, reproductive outcome of male carriers of chromosomal abnormalities, who

Genetics and Molecular Research 15 (4): gmr15048963 
accepted genetic counseling and psychological support, has been reported and its implications in genetic counseling have been discussed.

\section{MATERIAL AND METHODS}

\section{Patients}

Patients who sought pre-pregnancy counseling at the andrology outpatient's clinic of First Hospital of Jilin University, from January 2012 to December 2012, were consecutively recruited for this study. Total 2282 men were recruited, including 234 men with chromosomal abnormalities. Patients were considered to have oligozoospermia, if their last three semen samples had sperm counts less than $20 \times 10^{6}$ sperm $/ \mathrm{mL}$, severe oligozoospermia, if the sperm count was less than $5 \times 10^{6} \mathrm{sperm} / \mathrm{mL}$, or azoospermia, if no sperm were found in the ejaculate after centrifugation.

For all carriers of chromosomal abnormalities, we provided genetic counseling and psychological support. For each carrier, we introduced an appropriate way to conceive, applied assisted reproductive technology, assessed the success rate and the impact on the child's birth, etc. The carriers chose their own mode of reproduction. After 3 years, we performed a follow-up to determine the reproductive outcomes. The process is shown in Figure 1. The study protocol was approved by the Ethics Committee of First Hospital of Jilin University, Changchun, China, and all participants provided their written informed consent.

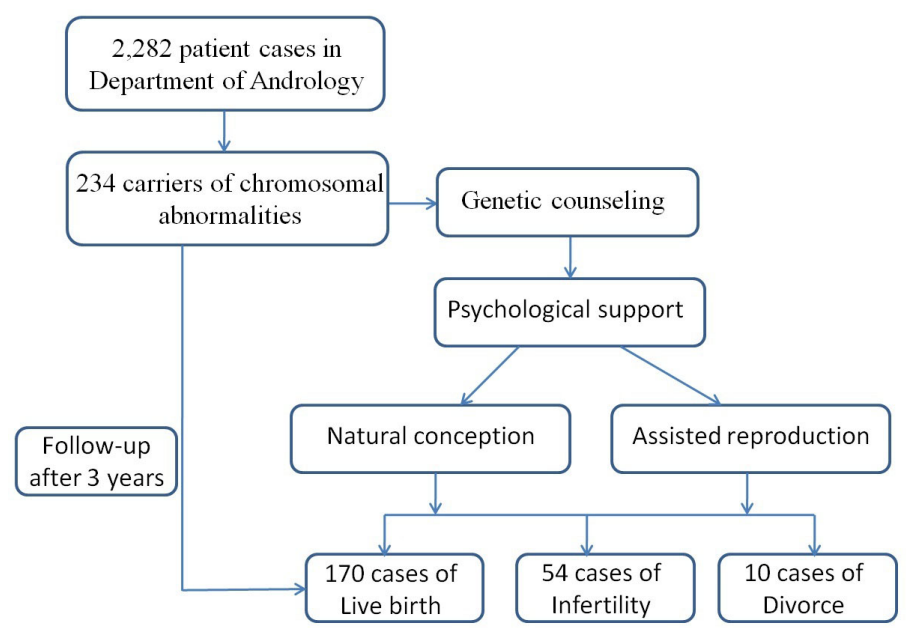

Figure 1. Flow chart of genetic counseling, psychological support, and follow-up of patients.

\section{Cytogenetic analysis}

Peripheral blood from all subjects was collected in sterile tubes containing $30 \mathrm{U} /$ $\mathrm{mL}$ heparin. $\mathrm{G}$ banding was performed using cultured peripheral blood lymphocytes. Lymphocytes were cultured in lymphocyte culture fluid (Yishengjun; Baidi Biological Pharmaceutical Co., Ltd., Guangzhou, China). The karyotypes of metaphase were analyzed for each subject (Wang et al., 2016). 


\section{Pregnancy outcome}

An elaborate questionnaire was completed, during follow-up visits, by the physician who attended the delivery. Follow-up questionnaire was provided to each carrier to collect their information, including pregnancy outcome, number of pregnancies, the way to conceive, number of abortions, assisted reproductions, number of live births, marital history, smoking history and frequency, alcohol consumption, occupational status, etc.

\section{RESULTS}

Among the 2282 male patients given pre-pregnancy counseling, 234 carriers (10.3\%) of chromosomal abnormalities were included. Karyotype and number of patients are presented in Table 1. Most cases, around 136 (58.1\%), were carriers of polymorphism.

Table 1. Karyotype and percentage of male carriers with various chromosomal abnormalities.

\begin{tabular}{l|c|c}
\hline Karyotype & Number of cases $(\mathrm{N}=234)$ & Percentage $(\%)$ \\
\hline Polymorphism & 136 & 58.1 \\
\hline $47, \mathrm{XXY}$ & 47 & 20.1 \\
\hline Balanced translocation & 22 & 9.4 \\
\hline Inv(9) & 13 & 5.6 \\
\hline Robertsonian translocation & 7 & 3.0 \\
\hline Inversion & 5 & 2.1 \\
\hline $47, \mathrm{XYY}$ & 2 & 0.8 \\
\hline Chimera & 2 & 0.8 \\
\hline
\end{tabular}

Among the 234 carriers of chromosomal abnormalities, clinical features showed normozoospermia, oligozoospermia, or azoospermia. Clinical features and reproductive outcomes of carriers exhibiting normozoospermia are presented in Table 2.

Table 2. Clinical features and reproductive outcomes of carriers of chromosomal abnormalities carrier exhibiting normozoospermia.

\begin{tabular}{l|c|c}
\hline Karyotype & No. of cases & Cases of live birth (\%) \\
\hline Polymorphism & 124 & $120(96.8)$ \\
\hline Balanced translocation & 21 & $18(85.7)$ \\
\hline Inv(9) & 13 & $13(100)$ \\
\hline Robertsonian translocation & 6 & $5(83.3)$ \\
\hline Inversion & 4 & $3(75)$ \\
\hline $47, X Y Y$ & 2 & $2(100)$ \\
\hline Total & 170 & $161(94.7)$ \\
\hline
\end{tabular}

Follow-up study showed that 10 carriers got divorced (Figure 1), including 2 carriers with polymorphism and 8 carriers with 47,XXY. Clinical features and reproductive outcomes of carriers exhibiting oligozoospermia or azoospermia are presented in Table 3.

Genetics and Molecular Research 15 (4): gmr15048963 
Table 3. Clinical features and reproductive outcomes of carriers of chromosomal abnormalities exhibiting oligozoospermia or azoospermia.

\begin{tabular}{l|l|c|c}
\hline Karyotype & Clinical features & No. of cases & Cases of live birth (\%) \\
\hline Polymorphism & Azoospermia & 10 & $1(10.0)$ \\
\hline \multirow{2}{*}{47, XXY } & Severe oligozoospermia & 5 & $4(80.0)$ \\
\cline { 2 - 4 } & Azoospermia & 34 & $2(5.9)$ \\
\hline Balanced translocation & Azoospermia & 1 & $0(0)$ \\
\hline Robertsonian translocation & Oligozoospermia & 1 & $1(100)$ \\
\hline Inversion & Azoospermia & 1 & $0(0)$ \\
\hline Chimera & Azoospermia & 2 & $0(0)$ \\
\hline Total & & 54 & $8(14.8)$ \\
\hline
\end{tabular}

\section{DISCUSSION}

Karyotype analysis is the most powerful and widely used application in reproductive medicine (Pasquier et al., 2016). Chromosomal abnormality is associated with infertility, and its incidence ranges from 2.1 to $15.5 \%$ in infertile men (Ananthapur et al., 2014). In this study, the detection rate of chromosomal abnormalities was $10.3 \%(234 / 2,282)$. Of these 234 carriers with chromosomal abnormalities, polymorphism was the most common abnormality (136 cases), followed by 47,XXY (47 cases) and balanced translocation ( 22 cases). Patients with Klinefelter syndrome (KS) are often diagnosed late (Grace, 2004). Most patients with KS were diagnosed because of concerns regarding their reproductive capacities (Zhang et al., 2015a). Carriers with balanced translocations may be phenotypically normal, but are associated with an increased risk of pregnancy loss (Godo et al., 2013). They are often diagnosed because they face reproductive problems.

The inability to conceive children is a stressful situation experienced by both individuals and couples all over the world (Cousineau and Domar, 2007). Therefore, infertile individuals with chromosomal abnormalities often receive double psychological distress. We explored reproductive outcomes of male carriers of chromosomal abnormalities, who accepted genetic counseling and psychological support. In this study, follow-up of the 170 carriers with normozoospermia showed that 161 cases $(94.7 \%)$ resulted in live births. For the carriers of polymorphisms, balanced translocations, inv(9), Robertsonian translocation, inversions, and 47,XYY, the cases of live birth were 120 (96.8\%), 18 (85.7\%), $13(100 \%), 5(83.3 \%), 3(75 \%)$, and $2(100 \%)$, respectively. Chromosomal polymorphisms usually occur in the pericentric heterochromatin on the long arms of chromosome 1,9, and 16 and in the heterochromatin on the short arms, satellites, or stalks of chromosome 13, 14, 15, 21, and 22 (Guo et al., 2012). Guo et al. (2012) reported that chromosomal polymorphisms seem to have deleterious effects on spermatogenesis, contributing to male infertility. Dong et al. (2014) reported that chromosomal polymorphisms do not have significantly lower live birth rates than non-carrier couples. For male translocation carriers, the success rates of natural pregnancies and live birth rates range from 30 to 70\% (Ozawa et al., 2008; Kochhar and Ghosh, 2013; Flynn et al., 2014). The limitation of this study was that it was a single center investigation with only 3 years of follow-up. A longer follow-up might have reported a higher live birth rate.

With the application of assisted reproductive technology, sperm can be obtained by testicular biopsy, testicular fine needle aspiration, and microdissection testicular sperm extraction. Hence, viable sperm were obtained from individual testicular tubules by biopsy, allowing for the patients with oligozoospermia or azoospermia to become fathers (Nieschlag, 
2013). In this study, follow-up of the 54 carriers with oligozoospermia or azoospermia showed that 8 cases resulted in live births. Among the 5 carriers of $47, \mathrm{XXY}$ and severe oligozoospermia, 4 cases resulted in live births. Among the 34 carriers of 47,XXY and azoospermia, 2 cases resulted in live births. In very few cases, KS men ejaculate spermatozoa and result in the birth of healthy children, following intracytoplasmic sperm injection (Zhang et al., 2015a). The technological development in testicular sperm extraction-intracytoplasmic sperm injection has assisted over $50 \% \mathrm{KS}$ patients, providing them with the opportunity of having biological children (Bar et al., 2014).

The present study explored reproductive outcome of male carriers of chromosomal abnormalities, who accepted genetic counseling and psychological support. Live birth rate among the carriers of polymorphisms and balanced translocations was higher than that reported in the literature (Ozawa et al., 2008; Pal et al., 2009; Flynn et al., 2014). These may be associated with psychological support. Hence, a multidisciplinary approach should be preferentially considered for carriers of chromosomal abnormalities.

In summary, $10.3 \%$ of the males enrolled in pre-pregnancy counseling were carriers of chromosomal abnormalities. Prospective study provided genetic counseling and psychological support to male carriers of chromosomal abnormalities. Followup of carriers with normozoospermia showed that $94.7 \%$ cases resulted in live births, whereas follow-up of carriers with oligozoospermia or azoospermia showed that $14.8 \%$ cases resulted in live births. Timely psychological support might be beneficial; therefore, multidisciplinary approach should be preferentially considered for the management of individuals with chromosomal abnormalities.

\section{Conflicts of interest}

The authors declare no conflict of interest.

\section{ACKNOWLEDGMENTS}

We would like to express our sincere gratitude to the staff of Genetics Laboratory, First Hospital of Jilin University for their excellent work.

\section{REFERENCES}

Ananthapur V, Avvari S, Veena K, Sujatha M, et al. (2014). Non-Robertsonian translocation t $(2 ; 11)$ is associated with infertility in an oligospermic man. Andrologia 46: 453-455. http://dx.doi.org/10.1111/and.12120

Bar G, Lunenfeld E and Levitas E (2014). [Klinefelter syndrome: genetic aspects, characteristics and reproduction-present and future]. Harefuah 153: 342-345, 366.

Cousineau TM and Domar AD (2007). Psychological impact of infertility. Best Pract. Res. Clin. Obstet. Gynaecol. 21: 293-308. http://dx.doi.org/10.1016/j.bpobgyn.2006.12.003

Dong Y, Li LL, Wang RX, Yu XW, et al. (2014). Reproductive outcomes in recurrent pregnancy loss associated with a parental carrier of chromosome abnormalities or polymorphisms. Genet. Mol. Res. 13: 2849-2856. http://dx.doi. org/10.4238/2014.January.17.4

Flynn H, Yan J, Saravelos SH and Li TC (2014). Comparison of reproductive outcome, including the pattern of loss, between couples with chromosomal abnormalities and those with unexplained repeated miscarriages. J. Obstet. Gynaecol. Res. 40: 109-116. http://dx.doi.org/10.1111/jog.12133

Gies I, Unuane D, Velkeniers B and De Schepper J (2014). Management of Klinefelter syndrome during transition. Eur. J. Endocrinol. 171: R67-R77.http://dx.doi.org/10.1530/EJE-14-0213

Genetics and Molecular Research 15 (4): gmr15048963 
Godo A, Blanco J, Vidal F and Anton E (2013). Accumulation of numerical and structural chromosome imbalances in spermatozoa from reciprocal translocation carriers. Hum. Reprod. 28: 840-849. http://dx.doi.org/10.1093/humrep/ des431

Grace RJ (2004). Klinefelter's syndrome: a late diagnosis. Lancet 364: 284. http://dx.doi.org/10.1016/S01406736(04)16679-8

Guo T, Qin Y, Gao X, Chen H, et al. (2012). The role of male chromosomal polymorphism played in spermatogenesis and the outcome of IVF/ICSI-ET treatment. Int. J. Androl. 35: 802-809. http://dx.doi.org/10.1111/j.1365-2605.2012.01284.x

Kochhar PK and Ghosh P (2013). Reproductive outcome of couples with recurrent miscarriage and balanced chromosomal abnormalities. J. Obstet. Gynaecol. Res. 39: 113-120.http://dx.doi.org/10.1111/j.1447-0756.2012.01905.x

Naasse Y, Charoute H, El Houate B, Elbekkay C, et al. (2015). Chromosomal abnormalities and Y chromosome microdeletions in infertile men from Morocco. BMC Urol. 15: 95. http://dx.doi.org/10.1186/s12894-015-0089-3

Nieschlag E (2013). Klinefelter syndrome: the commonest form of hypogonadism, but often overlooked or untreated. Dtsch. Arztebl. Int. 110: 347-353.

Ozawa N, Maruyama T, Nagashima T, Ono M, et al. (2008). Pregnancy outcomes of reciprocal translocation carriers who have a history of repeated pregnancy loss. Fertil. Steril. 90: 1301-1304. http://dx.doi.org/10.1016/j. fertnstert.2007.09.051

Pal S, Ma SO, Norhasimah M, Suhaida MA, et al. (2009). Chromosomal abnormalities and reproductive outcome in Malaysian couples with miscarriages. Singapore Med. J. 50: 1008-1012.

Pasquier L, Fradin M, Chérot E, Martin-Coignard D, et al. (2016). Karyotype is not dead (yet)! Eur. J. Med. Genet. 59: 11-15.http://dx.doi.org/10.1016/j.ejmg.2015.11.016

Peterson BD, Sejbaek CS, Pirritano M and Schmidt L (2014). Are severe depressive symptoms associated with infertilityrelated distress in individuals and their partners? Hum. Reprod. 29: 76-82. http://dx.doi.org/10.1093/humrep/det412

Spitczok von Brisinski I (2013). Klinefelter syndrome: the commonest form of hypogonadism, but often overlooked or untreated. Psychological and neurological problems. Dtsch. Arztebl. Int. 110: 675.

Wang HL, Wu B, Guo KM and Tian RH (2016). Psychological characteristics of and counseling for carriers of structural chromosome abnormalities. Genet. Mol. Res. 15: gmr.15028159.

Zhang HG, Wang RX, Li LL, Sun WT, et al. (2015c). Male carriers of balanced reciprocal translocations in Northeast China: sperm count, reproductive performance, and genetic counseling. Genet. Mol. Res. 14: 18792-18798. http:// dx.doi.org/10.4238/2015.December.28.28

Zhang M, Fan HT, Zheng HS, Zhang QS, et al. (2015a). Clinical characteristics of men with non-mosaic Klinefelter syndrome in northeastern China: implications for genetic counseling. Genet. Mol. Res. 14: 10897-10904. http:// dx.doi.org/10.4238/2015.September.9.27

Zhang M, Fan HT, Zhang QS, Wang XY, et al. (2015b). Genetic screening and evaluation for chromosomal abnormalities of infertile males in Jilin Province, China. Genet. Mol. Res. 14: 16178-16184.http://dx.doi.org/10.4238/2015. December.8.7

Genetics and Molecular Research 15 (4): gmr15048963 